\title{
Matrix polyester composite strengthened by munguba fiber (pseudobombax munguba)
} Amazon

\author{
Raimundo V. P. Lopes ${ }^{1 *}$, Jandecy C. Leite ${ }^{3 *}$, Roberto Tetsuo Fujiyama ${ }^{2}$, Marcus R. B. Afonso ${ }^{1}$, Eleodoro R. \\ Hermenegildo ${ }^{1}$ \\ ${ }^{1}$ Universidade Federal do Amazonas, Rua $1^{\circ}$ de maio N.5, Colônia. Benjamin Constant - AM - Brasil. CEP: 69630 - 000. Fone: \\ +55(97)34155677 / +55(92)993878930, valdanlopes@hotmail.com \\ ${ }^{2}$ Universidade Federal do Pará, Rua Augusto Correa 01 Guamá. Belém - Pará / Brasil. CEP: 66075-110. Fone: +55(91)32017435/ \\ $+55(91) 93147699$. \\ ${ }^{3}$ Instituto de Tecnologia e Educação Galileo da Amazônia - ITEGAM, Rua Via Láctea, 17, Morada do Sol. Aleixo. Manaus - Amazonas - Brasil. \\ CEP: 69060-085. Fone: +55 (92) 3584-6145 ou +55 (92) 3248-2646, jandecy.cabral@itegam.org.br
}

\begin{abstract}
The diversity of the species of the Amazon flora makes this region a large barn of plants suitable for the development of new polymeric composites. The article in question aimed to evaluate the mechanical properties of polyester composites reinforced by short fibers of Munguba from Amazon not only randomly arranged but also continuous and aligned fibers. For the evaluation of mechanical properties tensile test were used. The used method was qualitative and quantitative, comparing parameters obtained in composites developed by other literature. The results showed that for the investigated materials, the tensile strength increased with fiber length (5 mm - $9.61(\mathrm{MPa}) 10 \mathrm{~mm}-12.16(\mathrm{MPa})$ and $15 \mathrm{~mm}-19.91(\mathrm{MPa}))$ and when they remained better aligned, uniform and occupying a larger contact area in the matrix, the comportment was better; and composites with continuous and aligned fibers revealed the most satisfactory mechanical performance $(54,66 \mathrm{MPa})$.
\end{abstract}

Keywords: Munguba Fiber (Pseudobombax Munguba); Polymeric composite; Mechanical Properties; Tensile test.

\section{Matrix de composto fortalecidos de fibra de poliéster Munguba (Pseudobombax Munguba) Amazônia}

\section{RESUMO}

A diversidade das espécies da flora Amazônica torna esta região um grande celeiro de plantas adequadas para o desenvolvimento de novos materiais compósitos poliméricos. $\mathrm{O}$ artigo em questão teve como objetivo avaliar as propriedades mecânicas dos compósitos de poliéster reforçado por fibras curtas de Munguba da Amazônia organizadas não só de forma aleatória, mas também fibras contínuas e alinhadas. Para a avaliação das propriedades mecânicas foram utilizados teste de tração. O método utilizado foi qualitativo e quantitativo, comparando parâmetros obtidos em compósitos desenvolvidos por outras literaturas. Os resultados mostraram que, para os materiais investigados, a resistência à tração com o aumento do comprimento da fibra (5 mm - 9,61 (MPa) 10 mm - $12.16(\mathrm{MPa})$ e 15 milímetros - 19,91 (MPa)) e manteve-se melhor quando eles alinhados, uniforme e ocupando um área de contato maior na matriz, o comportamento foi melhor; e compósitos com fibras contínuas e alinhadas revelou o desempenho mecânico mais satisfatória (54,66MPa).

Palavras-chave: Munguba Fibra (Pseudobombax Munguba); Compostos poliméricos; Propriedades Mecânicas; Teste de tração.

\section{INTRODUÇÃO}

Nos últimos anos muitos esforços vêm sendo empregado por parte da indústria na busca por novos materiais com propriedades ecológicas e tecnológicas capazes de atender a novos requisitos tecnológicos e ambientais, em resposta à ineficiência no uso de materiais ditos convencionais e da dependência de matérias-primas provenientes de fontes não renováveis, as quais, já sinalizam indícios de escassez para as próximas décadas [1,2]. Estes e outros fatores têm influenciado o aumento da taxa média de crescimento mundial de biocompósito que entre 2003 e 2007 foi de $38 \%$ ao ano, nesse mesmo período, a taxa de crescimento anual na Europa foi de $48 \%$. Há, no entento, uma previsão de superação da capacidade de produção que era de 0,36 milhões de tonelada ocorrida no ano de 2007, 
Raimundo V. P. Lopes et al./ ITEGAM-JETIA Vol.01, Nº 02, pp.62-68. Junho, 2015.

para um aumento de 2,33 milhões de toneladas até 2013, e 3,45 milhoes de toneladas até 2020 [1].

Este cenário tem contribuído para uma maior participação de fibras provenientes de fontes renováveis na indútria devido: sua baixa densidade, baixo custo, estimula a geração de renda no campo, possuem boas propriedades mecânicas, físicas e químicas, amplamente distribuídas, baixo consumo de energia na produção e no processamento, disponíveis, moldáveis, maciez e abrasão reduzida, porosas, viscoelásticas, biodegradáveis, além de promove um processo seguro sem risco de contaminação do trabalhador [3,4,5]. Diante dessa perspectiva, no Brasil, a região Amazônica por ser uma região de clima tropical, possui uma diversidade de plantas propicias ao uso no desenvolvimento de novos compósitos como é o caso da fibra de Munguba (Pseudobombax munguba). Esta, junto com fibras de outras espécies de plantas, é utilizada no cotidiano por moradores da região amazônica na fabricação de cordas envira, usadas na confecção de cestos, redes, utensílios de pesca e de caça, objetos artesanais e outros.

A Mungubeira é uma espécie de árvore da família Malvaceae sensu latu, subgrupo das famílias Grewioideae, Byttnerioideae, Sterculioideae, Tilioideae, Dombeyoideae, Brownlowioideae, Helicteroideae, Malvoideae e Bombacoideae, típicas das várzeas amazônicas [6]. Sendo esta a única espécie do gênero encontrada em toda extensa planície inundada que se espalha desde a Amazônia peruana e colômbiana, até as planícies da foz do Rio Amazonas na amazonia brasileira [7].

Estudo com compósito de matriz poliéster biodegradável reforçada por fibras de curauá tratadas e in natura, nos comprimentos de 5, 10 e $20 \mathrm{~mm}$, os resultados de resistência à tração para compósitos com fibras de curauá in natura foram de 13,60 (MPa), 17,70 (MPa) e 14,50 (MPa). No caso de compósitos com fibras da curauá tratadas quimicamente, os valores de resistência a tração foram de 14,80 (MPa), 19,30 (MPa) e 22,2 (MPa), em ambos os casos, o desempenho mecânico obedeceu respectivamente ao tamanho das fibras [8]. Compósitos de resina poliéster de alta densidade reforçados por polpa de fibras de bambu alcançaram no ensaio de tração 17,5 (MPa), cujos resultados mais satisfatórios foram obtidos mediante o uso de compatibilizantes [9]. Compósitos de Poliuretano reforçados por fibras de curauá nas proporções de 5, 10 e $20 \%$, alcançaram no ensaio resistência à tração 14,7 (MPa), com desempenho mais satisfatório nos compósitos com proporções de 5\% fibra curaúa [10].

Em compósitos híbridos de poliéster insaturado reforçado por fibras curtas de sisal e seda de 1,2 e $3 \mathrm{~cm}$, os resultados mecânicos no ensaio de tração com base nos comprimento das fibras para compósitos híbridos com fibras in natura, foi de 16,57 (MPa), 18,94 (MPa) e 17,54 (MPa). No caso dos compósitos híbridos com fibras tratadas quimicamente, o resultado no ensaio de tração foi de 20,86 (MPa), 23,60 (MPa) e 21,31(MPa), sendo os compósitos tratados quimicamente, os que apresentaram melhores valores mecânico [11]. No caso de compósitos puros de matriz epóxi com fibras de banana e sisal de $15 \mathrm{~mm}$, o resultado do ensaio de tração para compósitos com fibras de banana foi de 16,12 (MPa) e para os materiais compósitos com fibra de sisal foi de 21,20 (MPa) [12].
A pesquisa teve como objetivo, a otimização das propriedades de compósitos de matriz poliéster tereftalico reforçados por fibras de Munguba (Pseudobombax Munguba), em função do comprimento $(\mathrm{mm})$, determinação da massa especifica (\%) e aspectos superficiais das fibras. Com o auxilio de microscopia eletrônica de varredura - MEV analisou-se a influência do comprimento e da massa especifica das fibras utilizadas, e seus efeitos nas propriedades mecânicas dos compósitos, mediante a análise da fratura a partir do ensaio de tração. E cujas propriedades mecânicas foram comparadas as de compósitos reforçados por outras fibras vegetais.

\section{MATERIAIS, MÉTODOS E APLICAÇÃO}

Resina Poliéster: a resina utilizada foi a poliéster tereftálica insaturada e pré-acelerada, fabricada pela Royal Polímeros sob a denominação comercial de Denverpoly 754. O agente de cura utilizado foi o peróxido de MEK (Butanox M-50), na proporção de $0,33 \%(\mathrm{v} / \mathrm{v})$. A resina foi adquirida já préacelerada com naftenato de cobalto (CoNap) na proporção mássica de $0,15 \%$. A proporção de agente de cura/resina de $0,33 \%(\mathrm{v} / \mathrm{v})$, utilizada tende a melhorar o desempenho mecânico do compósito [13].

Fibra de Munguba: as fibras de Munguba foram extraídas de forma manual da parte intermediária e topo de árvores adultas nativas, com o uso de facões, na região de várzea do Curarí, situada às margens do rio Amazonas, no Município do Careiro da Várzea a 25,74 Km de Manaus/AM.

$\mathrm{O}$ desfibramento e a separação das fibras foram realizados manualmente, em seguida, cortadas com estiletes e tesouras manuais em que se obtiveram diferentes comprimentos de fibras com $5 \mathrm{~mm}, 10 \mathrm{~mm}$ e $15 \mathrm{~mm}$, sendo este um procedimento consideravelmente simples, uma vez que as fibras já haviam permanecido submersas em água em um recipiente por 48 horas, cujo intuito era torna-las maleáveis e de fácil manuseio. Tal processo foi realizado nas dependências do Laboratório de Materiais Compósitos da Faculdade de Engenharia Mecânica da Universidade Federal do Pará - UFPA.

Confecção dos copos de prova: a confecção dos corpos de prova do compósito consistiu em um processo bastante simplificado, mediante moldagem manual, utilizando moldes de silicone sem o uso de desmoldante e sem pressão. No caso do trabalho em questão, usou-se resina poliéster reforçada por fibras curtas dispostas aleatoriamente nos comprimento de 5, 10 e 15 $\mathrm{mm}$, cujas proporções mássicas obedeceram respectivamente $10,08 \%, 10,58 \%$ e $5,22 \%$.

A fabricação dos compósitos ocorreu mediante matriz previamente otimizada, para a fabricação de compósito com fibras curtas, misturando as quantidades pré-pesadas de resina, agente de cura e, no caso do trabalho em questão, diferentes porcentagens e tamanhos de fibras de Munguba em um Becker, com mistura homogeneizada num período de 5 (cinco) minutos e vazada a temperatura ambiente, em moldes de silicone.

A fração mássica de cada tipo de reforço utilizada na confecção dos compósitos teve como base a capacidade volumétrica do molde em acomodar o reforço sem pressão ou compactação e na ausência da matriz. Para cada tipo de reforço por as fibra de Munguba nas proporções de 5, 10 e $15 \mathrm{~mm}$, os 
moldes de silicone foram enchidos com reforço até o limite de sua capacidade volumétrica, sem aplicação de pressão ou vibração mecânica. Na sequência, cada reforço foi devidamente pesado, e o valor da massa obtida, convertida em fração mássica, sendo esta estabelecida como sendo a referência de incorporação e trabalhabilidade para a fabricação de compósitos sem pressão. A partir desse valor de referência foi possível estabelecer as proporções a serem utilizadas nos compósitos de reforço por diferentes quantidades de fibra de Mungubas.

Uma vez estabelecidos os valores de referência para a fração mássica, deu-se início a confecção dos copos de prova para a avaliação da trabalhabilidade da mistura sobre o estado liquido, em que se avaliou sua moldabilidade e a molhabilidade da matriz sobre os reforços, sendo retificados os valores de referência. A partir desse procedimento pôde se estabelecer as proporções do compósito, os quais foram repetidas duas vezes para cada comprimentos das fibras Munguba previamente confeccionadas e proporção de fração mássica estabelecida (5 $\mathrm{mm}-10,08 \%$, $10 \mathrm{~mm}$ - 10,58\% e $15 \mathrm{~mm}$ - 5,22\%), sendo utilizado $0,33 \%(\mathrm{v} / \mathrm{v})$ de gente de cura para cada série.

Ensaios de tração do material compósito: Os ensaios de tração ocorreram em conformidade com a norma ASTM D 638M. A confecção dos compósitos para o ensaio de tração ocorreu mediante o uso de moldes de silicone com um número de 24 (vinte e quatro) corpos de prova com matriz reforçada por fibras curtas de Munguba. Os quais foram ensaiados em uma máquina universal modelo KRATOS MKCA-KE, com sistema de aquisição de dados, com célula de carga de $5 \mathrm{KN}$, adotando velocidade de $5 \mathrm{~mm} / \mathrm{min}$ e comprimento útil para medição entre garras de $60 \mathrm{~mm}$.

Análises da superfície da fratura dos corpos de prova: A análise da superfície da fratura dos compósitos ocorreu mediante Microscopia Eletrônica de Varredura - MEV, em que se analisou a morfológica da superfície da fratura e os principais mecanismos de falha de cada compósito ensaiado.

\section{RESULTADOS E DISCUSSÕES}

Caracterização das fibras de Munguba: as fibras de Munguba in natura foram caracterizadas quanto ao comprimento (mm), determinação da massa especifica (\%) e aspectos superficiais. A análise microestrutural ocorreu mediante Microscopia Eletrônica de Varredura - MEV, em que se verificou $\mathrm{o}$ aspecto superficial e a seção transversal das fibras, além de amostras embutidas na matriz de poliéster tereftálico. A Figura 1 mostra por meio de MEV, o ângulo longitudinal e frontal da fibra, onde é possível observar microcavidade em sua superfície

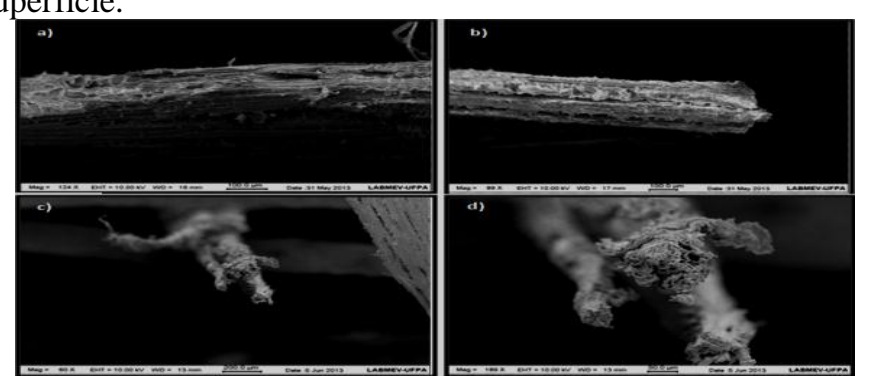

Figura 1: Imagem de MEV da fibra de Munguba in natura: a) ângulo longitudinal, b) microcavidades na superfície da fibra e c) e d) ângulo frontal da fibra.

O valor médio da massa especifica das fibras de Munguba ocorreu em concomitância ao comprimento médio das fibras, sendo estas pesadas em balança de precisão modelo Marte, da Marte Balança e Aparelhos de Precisão LTDA. A determinação do teor de umidade/absorção máxima das fibras se deu mediante a secagem destas num período de 15 minutos em forno elétrico modelo Layr Midy Inox, onde se determinou o teor base de umidade em três (3) amostras de fibras, com massa total de 34,50 gramas. As fibras foram inicialmente pesadas e secas até a massa constante.

Características mecânicas e microestrutural dos materiais compósitos: a fabricação, acabamento, dimensionamento e marcação dos compósitos com fibra de Munguba ocorreram conformidade com especificações das normas ASTM D 638 e ASTM D 3039M para o ensaio mecânico de tração.

Materiais compósitos com fibras de Munguba de $5 \mathrm{~mm}$ : Os resultados obtidos no ensaio de tração para os compósitos de poliéster reforçado por fibras de Munguba nos comprimentos de $5 \mathrm{~mm}$ mostrados na Tabela 1 revelaram valores relativamente baixos no limite de resistência a tração.

Tabela 1: Resultados dos ensaios de tração de compósitos poliéster tereftálico reforçados por fibras de Munguba de $5 \mathrm{~mm}$.

\begin{tabular}{|c|c|c|c|c|c|}
\hline & Força (kN) & $\begin{array}{c}\text { Desloca. } \\
(\mathrm{mm})\end{array}$ & $\begin{array}{c}\text { Tensão Max } \\
(\mathrm{MPa})\end{array}$ & $\begin{array}{c}\text { Módulo de Elasticidade } \\
(\mathrm{GPa})\end{array}$ & $\begin{array}{c}\text { Deformação } \\
(\mathrm{mm} / \mathrm{mm})\end{array}$ \\
\hline Média & 0,32 & 2,01 & 9,61 & 0,29 & 0,03 \\
\hline Desv. & 0,06 & 0,23 & 2,17 & 0,04 & 0,00 \\
\hline
\end{tabular}

A Figura 2 fornece uma melhor compreensão do esforço mecânico aplicado, em que se constatam vários ciclos de rompimento no compósito durante o ensaio de tração.

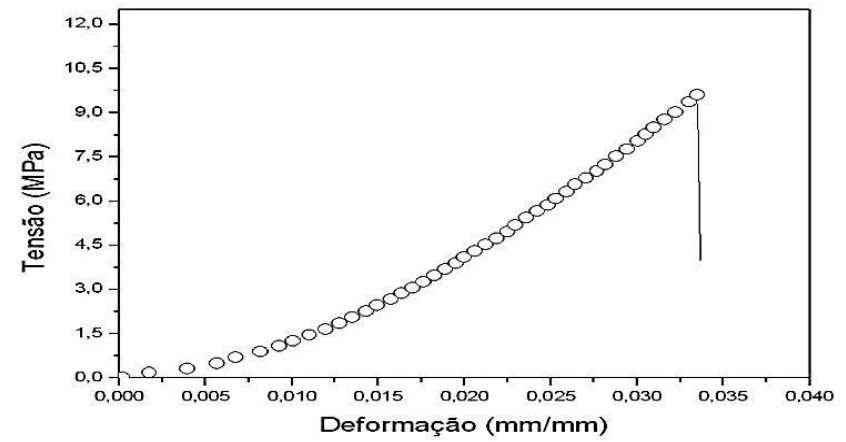

Figura 2: Tensão (MPa) versus Deformação $(\mathrm{mm} / \mathrm{mm})$ de corpos de prova de com fibras de $5 \mathrm{~mm}$.

A análise microetrutural de compósitos com fibras de Munguba de $5 \mathrm{~mm}$ oferece uma melhor compreensão do comportamento interno do compósito, considerando na respectiva análise, o comportamento das fibras de reforço, formação de trincas, crateras por deslocamento de fibras, como 
forma de se obter resposta para o baixo valor mecânico obtido no ensaio de tração com esse tipo de compósito.

A Figura 3 mostra múltiplas crateras e fibras expostas em um plano definido, resultado do arrancamento (pull out) de fibra da matriz, consequência da baixa adesão fibra-matriz em compósito com fibras de $5 \mathrm{~mm}$, sendo o tamanho das fibras neste caso, um fator limitante considerando que estas passavam a ocupar uma área de contato menor na região da matriz. Além da presença de fibras em direção transversal com desalinhamento e não uniformidade, e fibras rompidas com o esforço mecânico aplicado. a)

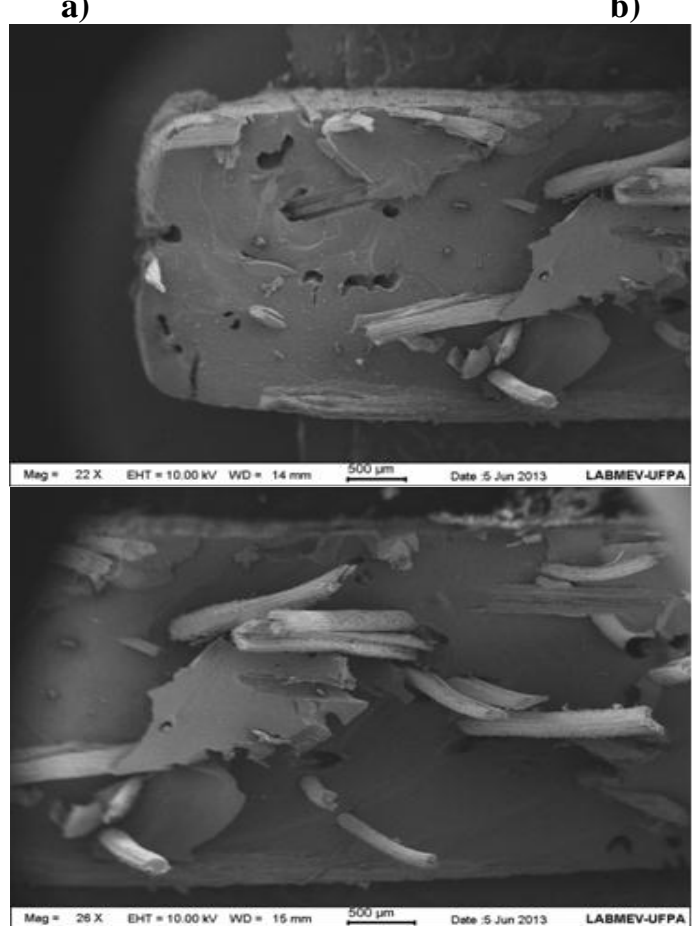

Figura 3: Superfície de fratura de compósito poliéster tereftalico reforçado por fibras de Munguba de $5 \mathrm{~mm}$ : a) e b) múltiplas crateras provocadas pelo arrancamento de fibras (pull out) no compósito durante o esforço mecânico aplicado.

Materiais compósitos com fibras de Munguba de 10 mm: Os resultados obtidos no ensaio de tração para os compósitos de poliéster reforçados por fibras de Munguba de 10 $\mathrm{mm}$, mostrados na Tabela 2 alcançaram um maior valor médio no limite de resistência à tração, com uma diferença de 2,55 (MPa) em comparação aos reforçados por fibras de $5 \mathrm{~mm}$.

Tabela 2: Resultado do ensaio de tração de compósitos poliéster tereftálico reforçado por fibras de Munguba nas proporções de $10 \mathrm{~mm}$.

\begin{tabular}{|c|c|c|c|c|c|}
\hline & Força (KN) & $\begin{array}{c}\text { Desloca. } \\
(\mathrm{mm})\end{array}$ & Tensão Max (MPa) & $\begin{array}{c}\text { Módulo de } \\
\text { Elasticidade (GPa) }\end{array}$ & $\begin{array}{c}\text { Deformação } \\
(\mathrm{mm} / \mathrm{mm})\end{array}$ \\
\hline Média & 0,40 & 2,69 & 12,16 & 0,27 & 0,04 \\
\hline Desv. & 0,09 & 0,44 & 2,46 & 0,03 & 0,01 \\
\hline
\end{tabular}

A Figura 4 mostra o gráfico Tensão (MPa) versus Deformação $(\mathrm{mm})$ dos corpos de prova após o ensaio de tração para compósito de poliéster reforçado por fibras de munguba de $10 \mathrm{~mm}$ de comprimento, em que se obteve uma diferença de $21 \%$ (MPa), em comparação aos com fibras de $5 \mathrm{~mm}$.

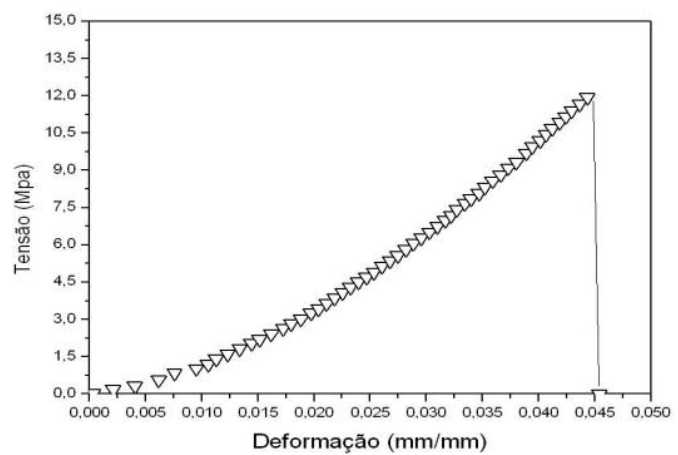

Figura 4: Tensão (MPa) versus Deformação $(\mathrm{mm} / \mathrm{mm})$ de corpos de prova de com fibras de $10 \mathrm{~mm}$.

$\mathrm{Na}$ análise interna da fratura de compósito com fibras de Munguba de $10 \mathrm{~mm}$ por meio de MEV foi constatado uma maior resistência mecânica dos compósitos, considerando a integridade das fibras e da superfície do mesmo, em comparação aos com fibras de $5 \mathrm{~mm}$. Constata-se ainda, a ocorrência de arrancamento (pull out) de fibras e crateras disformes provocadas pela baixa interação das fibras na matriz como mostra a Figura 5.

a)

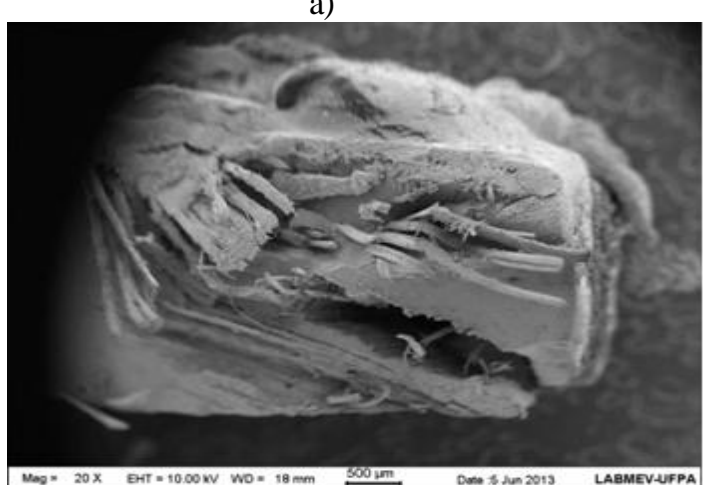

b)

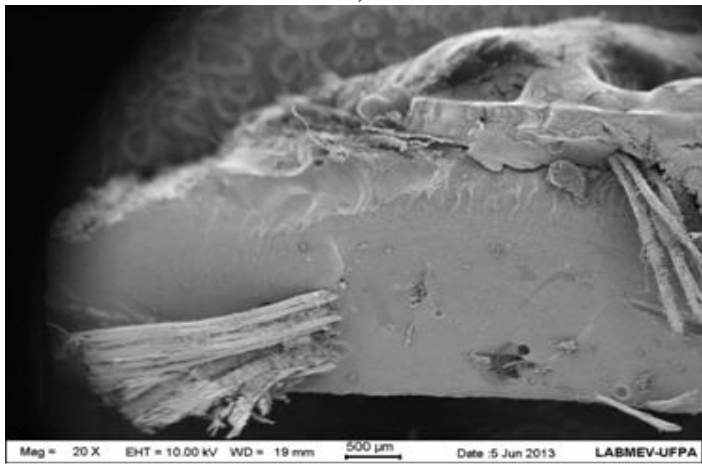

Figura 5: Superfície de fratura de compósito poliéster tereftalico reforçado por fibras de Munguba de $10 \mathrm{~mm}$, cuja seta preta indica a ocorrência de crateras disforme e a seta branca o arrancamento (pull out) das fibras durante o esforço mecânico no compósito. 
Materiais compósitos com fibras de Munguba de 15 mm: A Tabela 3 apresenta os resultados obtidos nos ensaios de resistência à tração mecânica dos compósitos reforçados por fibras de $15 \mathrm{~mm}$. Fornecendo uma melhor compreensão do esforço mecânico aplicado e suas proporcionalidades nos compósitos, em que se obteve uma diferença de $52 \%(\mathrm{MPa})$ em comparação com os reforçados por fibras de $5 \mathrm{~mm}$ e, $39 \%$ (MPa) em comparação com os reforçados por fibras de $10 \mathrm{~mm}$.

Tabela 3: Resultados dos ensaios de tração dos compósitos de poliéster tereftálico reforçado por fibras de Munguba nas proporções de $15 \mathrm{~mm}$.

\begin{tabular}{|c|c|c|c|c|c|}
\hline & Força $(\mathrm{kN})$ & $\begin{array}{c}\text { Desloca. } \\
(\mathrm{mm})\end{array}$ & $\begin{array}{c}\text { Tensão Max } \\
(\mathrm{MPa})\end{array}$ & $\begin{array}{c}\text { Módulo de } \\
\text { Elasticidade (GPa) }\end{array}$ & Deformaç̃ão (mm/mm) \\
\hline Média & 0,63 & 3,03 & 19,91 & 0,39 & 0,05 \\
\hline Desv. & 0,09 & 0,13 & 2,82 & 0,06 & 0,00 \\
\hline
\end{tabular}

A Figura 6 mostra o gráfico Tensão (MPa) versus Deformação (mm) dos corpos de prova após o ensaio de tração para o compósito de poliéster reforçado por fibras de munguba de 15 mm de comprimento.

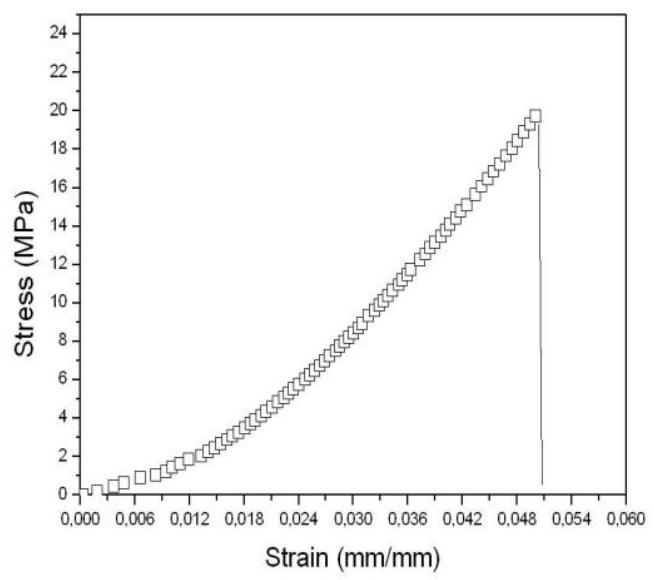

Figura 6: Força $(\mathrm{N})$ versus alongamento $(\mathrm{mm})$ com corpos de prova de $15 \mathrm{~mm}$.

Na caracterização microetrutural interna do compósito com fibras de $15 \mathrm{~mm}$, como mostra a Figura 7 foi evidenciado uma drástica redução de crateras e arrancamento de fibras (pull out) na matriz, com ocorrência de trincas (resultado da concentração de tensões) e bolhas conforme as setas pretas, com as fibras se mantendo integras durante esforço mecânico aplicado. Tal fato foi influenciado pelo comprimento das fibras, uma vez que estas ocupavam uma área de contato maior na matriz, além de estarem melhor alinhadas e uniformes, o que promoveu, por consequência, melhor interação interfacial fibramatriz facilitando a transferência de carga da matriz para as fibras, garantindo dessa forma, resultados mecânicos mais satisfatórios para este tipo de compósito.

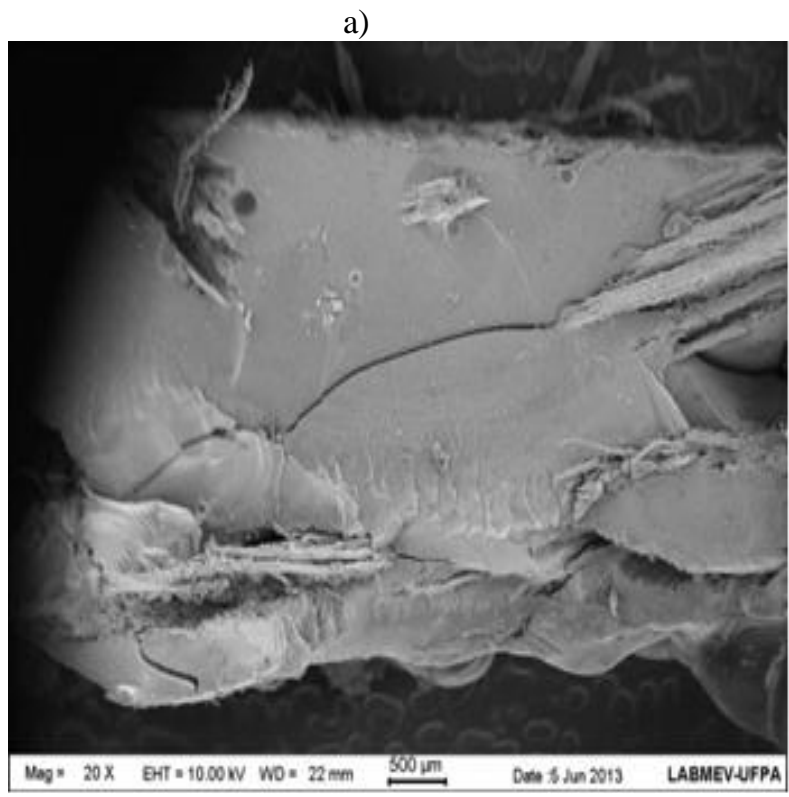

b)

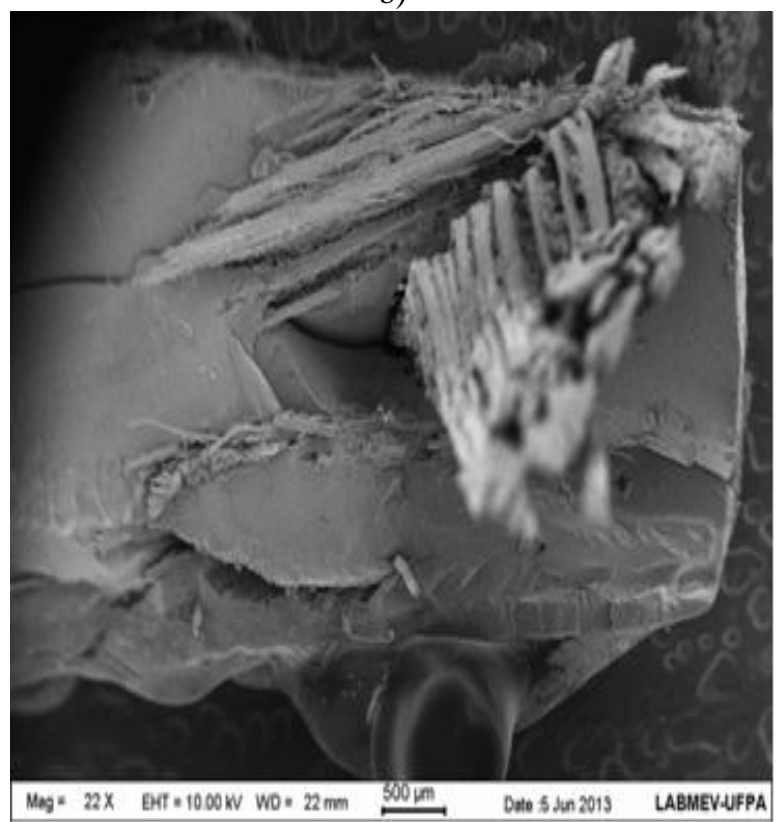

Figura 7: Superfície de fratura de compósito poliéster tereftalico reforçado por fibras de Munguba de $15 \mathrm{~mm}$ : a) tricas resultantes da concentração de tensão no compósito e b) rompimento e arranchamento (pull out) de fibras durante o esforço mecânico aplicado no compósito.

Materiais compósitos com fibras de munguba continuas $\boldsymbol{e}$ alinhadas: Para o ensaio de tração com esse tipo compósito foram utilizados 08 (oito) corpos de prova conforme a norma ASTM D 3039M. Os valores mecânicos obtidos no ensaio mecânico em questão revelaram-se superiores em comparação aos obtidos em compósitos reforçados por fibras curtas, o que pode ser justificado pelo melhor alinhamento das fibras na direção do carregamento com esse tipo de compósito, o que facilitou a transferência de carga matriz/fibra. 
Raimundo V. P. Lopes et al./ ITEGAM-JETIA Vol.01, N 02, pp.62-68. Junho, 2015.

Tabela 4: Desempenho mecânico de compósitos com fibras continuas e alinhadas.

\begin{tabular}{|c|c|c|c|c|c|}
\hline & $\begin{array}{c}\text { Força } \\
(\mathrm{kN})\end{array}$ & $\begin{array}{c}\text { Desloca. } \\
(\mathrm{mm})\end{array}$ & $\begin{array}{c}\text { Tensão Max } \\
(\mathrm{MPa})\end{array}$ & $\begin{array}{c}\text { Módulo de } \\
\text { Elasticidade }\end{array}$ & $\begin{array}{c}\text { Deformação } \\
(\mathrm{mm} / \mathrm{mm})\end{array}$ \\
\hline Média & 2,54 & 3,74 & 54,66 & 1,97 & 0,022 \\
\hline Desv. Pad. & 0,31 & 0,30 & 5,82 & 0,19 & 0,003 \\
\hline
\end{tabular}

A Figura 8 mostra o gráfico Tensão (MPa) versus Deformação $(\mathrm{mm})$ de compósito com fibras continuas e alinhadas.

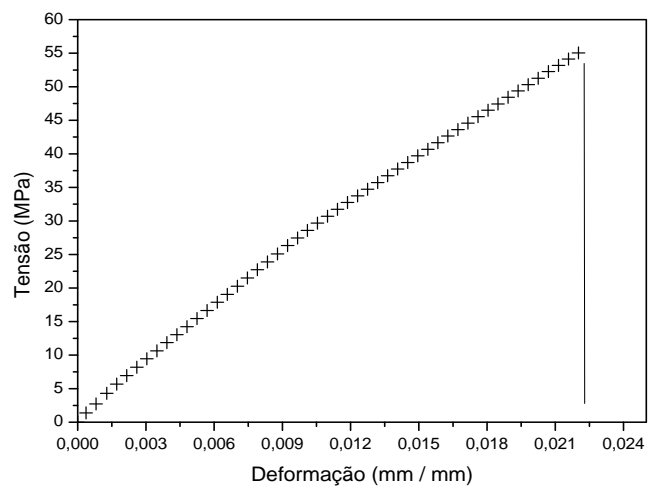

Figura 8: Material compósito de poliéster reforçado por fibras de munguba contínua e alinhada.

Desempenho mecânico dos compósitos de poliéster reforçados por fibras de munguba de $5 \mathrm{~mm}, 10 \mathrm{~mm}$ e $15 \mathrm{~mm} e$ os com fibras contínuas $e$ alinhadas: A Figura 9 mostra o gráfico Tensão (MPa) versus Deformação $(\mathrm{mm})$ de materiais compósitos de matriz poliéster reforçado por fibras de $5 \mathrm{~mm}, 10$ $\mathrm{mm}$ e $15 \mathrm{~mm}$ de comprimento e os com fibras contínuas e alinhadas, em que se contata um melhor desempenho mecânico no ensaio de tração nos compósitos com fibras contínuas e alinhadas, sento tal resultado influenciado pelo melhor alinhamento das fibras nesse tipo de compósito. Alinhamento de fibras em direção de carregamento tende a exercer influência nas propriedades mecânicas do material compósito.

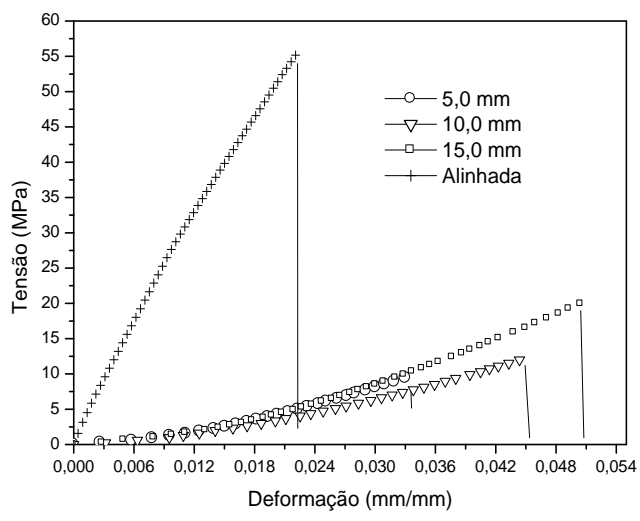

Figura 9: Material compósito de poliéster reforçado por fibras de munguba de $5 \mathrm{~mm}, 10 \mathrm{~mm}$ e $15 \mathrm{~mm}$ de comprimento e com fibras contínuas e alinhadas.
Conforme a determinação do comprimento (mm), massa especifica (\%) e aspectos superficiais das fibras nas propriedades do compósito com resina poliéster tereftalico, reforçado por fibras curtas de mungubas disposta aleatoriamente e continuas e alinhadas, os resultados indicaram que: $\mathrm{O}$ aumento nas propriedades mecânicas dos compósitos no ensaio de resistência a tração foram respectivos aos comprimentos das fibras e, a medida que estas se mantinham melhor alinhadas, uniformes e passavam a ocupar uma área de contato maior na matriz conforme MEV. Promovendo melhor transferência de carga da matriz para as fibras.

Vale ressaltar, no entanto que os aspectos mecânicos de materiais compósitos são influenciados por fatores que vão além dos fatores que se aplicam a pesquisa em questão. Tais tendências também foram acompanhadas pela Força Máxima $(\mathrm{kN})$. Sendo que as porcentagens mássicas (\%) das fibras não se revelaram um fator preponderante nas propriedades mecânicas dos compósitos.

A análise microestrutural interna da fratura do compósito obtida mediante MEV revelaram na superfície da matriz de compósito com fibras de $15 \mathrm{~mm}$, boa adesão entre os constituintes, com baixo índice de arrancamento (pull out) e formação de crateras, além de um melhor alinhamento e uniformidade das fibras. Conferindo a este tipo de compósito, melhor desempenho mecânico, quando comparado aos com fibras de 5 e $10 \mathrm{~mm}$.

$\mathrm{Na}$ Tabela 5 torna-se perceptível a superação e, em outros casos, uma aproximação dos valores obtidos no ensaio mecânico de tração de compósito reforçado por fibras curtas de munguba dispostas aleatoriamente, em comparação aos obtidos de outros estudos $[8,9,10,11,12]$.

Tabela 5: resultados comparativos (\%) de compósitos usando fibra de Munguba com os de outras fibras.

\begin{tabular}{|c|c|c|c|}
\hline Matriz & Reforço & $\begin{array}{c}\text { Limite de } \\
\text { Resistência }\end{array}$ & $\begin{array}{c}\text { Diferença } \\
\text { em \% em }\end{array}$ \\
\hline Poliéster & Munguba & $\mathbf{1 9 , 9 1}$ & - \\
\hline $\begin{array}{c}\text { Poliéster } \\
\text { biodegradável }\end{array}$ & Curauá & 17,70 & $-11 \%$ \\
\hline $\begin{array}{c}\text { Poliéster de alta } \\
\text { densidade }\end{array}$ & Bambu & 17,5 & $-12 \%$ \\
\hline Poliuretano & Curauá & 14,7 & $-26 \%$ \\
\hline Poliéster & Sisal/Seda & 18,94 & $-5 \%$ \\
\hline Epóxi & Banana & 16,39 & $-17 \%$ \\
\hline
\end{tabular}

\section{CONCLUSÕES}

A fabricação de compósito de poliéster com fibra de Munguba ocorreu mediante um processo consideravelmente simples, com um menor nível possível de processamento nas etapas produtivas, em que se obteve um material tecnicamente viável. Tais resultados, quando comparados com os de outros trabalhos, levam a inferir que o compósito em questão, possui propriedade mecânica viável no contexto do uso e 
desenvolvimento de materiais compósitos poliméricos. Ao mesmo tempo contribui com as discursões em torno da viabilidade no uso de fibras vegetais amazônicas, para o desenvolvimento de novos compósitos polimérico, em prol da pesquisa cientifica e da sustentabilidade.

\section{AGRADECIMENTOS}

A Universidade Federal do Amazonas (UFAM), Universidade Federal do Pará (UFPA), Fundação de Amparo à Pesquisa do Estado do Amazonas (FAPEAM) e Instituto de Tecnologia Galileo da Amazônia (ITEGAM), pelo apoio na realização dessa pesquisa.

\section{REFERÊNCIAS BIBLIOGRÁFICAS}

[1] Faruk, O. et al., 2012. Biocomposites reinforced with natural fibers: $2000-2010$. Progress in Polymer Science. Toronto. n. 37, p.1552-1596.

[2] Kanter, J. E. U., 2010, Faces Shortages of Key Minerals. The New York Times, New York, 16 Jun.

[3] Monteiro, S. N. et al, 2012, (Thermogravimetric Stability Behavior of Less Common Lignocellulosic Fibers. Journal of Materials Research and Technology. Rio de Janeiro. v.1, n.3, rev. de artig. p.189-199.

[4] Levy Neto, F. L and Pardini, L. C., 2006, Compósitos Estruturais: Ciência e Tecnologia. São Paulo: Edgard Blucher Ltda.

[5] Prasad, A. V. Ratna and Rao, K. Mohana., 2011, Mechanical properties of natural fibre reinforced polyester composites: Jowar, sisal and bamboo. Materials and Design. 32, p. 4658 4663.

[6] Menicucci, T. A., 2007, Filogeografia e estrutura genética de populações da mungubeira (pseudobombax munguba (mart.\&zucc.) dugand,malvaceae - bombacoideae) na Amazônia brasileira. 2007. Dissertação (Mestrado em Biologia Tropical e Recursos Naturais), Instituto Nacional de Pesquisas da Amazônia e Universidade Federal do Amazonas, Manaus/AM.

[7] Gribel, R. and Abbott, R. J., 1995. Genetics of cytosolic phosphoglucose isomerase (PGI) variation in the Amazonian tree Pseudobombax munguba (Bombacaceae). The Genetical Society of Great Britain. Manaus. n. 76, p. 531-538.

[8] Harnnecker, F. et al, 2012, Biodegradable Polyester-Based Blend Reinforçed with Curauá Fiber: Thermal, Mechanical and Biodegradation Behaviour. Journal of Polymers and the Environment. vol. 20, p. 237-244.
[9] Liu, H. et al, 2008, Compatibilizing and toughening bamboo flour-filled HDPE composites: Mechanical properties and morphologies. Composites: Part A, n. 39, p. 1891-1900.

[10] Mothé, C. G. and ARAÚJO, C. R., 2004, Caracterização térmica e mecânica de compósitos de poliuretano com fibras de Curauá. Polímeros: Ciência e Tecnologia. v. 14, n. 4, p.274278.

[11] Khanam, P. N. et al, 2007, Tensile, Flexural and Compressive Properties of Sisal/Silk Hybrid Composites. Journal of Reinforced Plastics and Composites, v. 26, n. 10. [12] Venkateshwaran, N. and Elayaperumal, A., 2011, Modeling and evaluation of tensile properties of randomly oriented banana/epoxy composite. Journal of Reinforced Plastics and Compósite. n. 30, p. 1957-1967.

[13] Rodrigues, J. S., 2010, Análise fractográfica de compósitos de matriz poliéster e resíduos da indústria madeireira. VI Congresso Nacional de Engenharia Mecânica CONEM, 18 a 21 de Agosto. Campina Grande/PB. 